\title{
Burden of prediabetes, undiagnosed, and poorly or potentially sub-controlled diabetes: Lolland-Falster health study
}

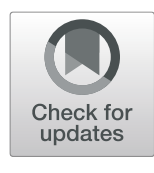

\author{
Neda Esmailzadeh Bruun-Rasmussen 1* (D), George Napolitano², Allan Kofoed-Enevoldsen ${ }^{3}$, Stig Egil Bojesen ${ }^{4}$, \\ Christina Ellervik ${ }^{5,6}$, Knud Rasmussen ${ }^{5}$, Randi Jepsen ${ }^{1}$ and Elsebeth Lynge ${ }^{1}$
}

\begin{abstract}
Background: This study aimed to investigate prevalence and risk factors for prediabetes, undiagnosed diabetes mellitus, poorly and potentially sub-controlled diabetes in a rural-provincial general adult population in Denmark.

Methods: Using cross-sectional data from the Lolland-Falster Health Study, we examined a total of 10,895 individuals aged 20 years and above.

Results: Prevalence of prediabetes was 5.8\% (men: 6.1\%; women: 5.5\%); of undiagnosed diabetes $0.8 \%$ (men: 1.0\%; women: $0.5 \%$ ); of poorly controlled diabetes $1.2 \%$ (men: $1.5 \%$; women: $0.8 \%$ ); and of potentially sub-controlled diabetes 2\% (men: 3.0\%; women: 1.3\%). In total, $9.8 \%$ of all participants had a diabetes-related condition in need of intervention; men at a higher risk than women; RR 1.41 (95\% Cl 1.26-1.58); person aged + 60 years more than younger; RR 2.66 (95\% Cl 2.34-3.01); obese more than normal weight person, RR 4.51 (95\% Cl 3.79-5.38); smokers more than non-smokers, RR 1.38 (95\% Cl 1.19-1.62); persons with self-reported poor health perception more than those with good, RR 2.59 (95\% Cl 2.13-3.15); low leisure time physical activity more than those with high, RR 2.64 (95\% Cl 2.17-3.22); and persons with self-reported hypertension more than those without, RR 3.28 (95\% Cl 2.93-3.68).

Conclusions: In the Lolland-Falster Health Study, nearly $10 \%$ of participants had prediabetes, undiagnosed diabetes, poorly controlled, or potentially sub-controlled diabetes. The risk of these conditions was more than doubled in persons with self-reported poor health perception, self-reported hypertension, low leisure time physical activity, or measured obesity, and a large proportion of people with diabetes-related conditions in need of intervention can therefore be identified relatively easily.
\end{abstract}

Keywords: Diabetes mellitus, Prediabetes, Undiagnosed diabetes, Glycaemic control, Lolland-Falster health study

\section{Background}

Diabetes mellitus (DM) is a metabolic disease characterized by high blood glucose levels resulting from defects in insulin secretion, insulin action, or both [1]. It is one of the most prevalent chronic diseases worldwide affecting more than 451 million adults in 2017 with a

\footnotetext{
* Correspondence: neebruun@gmail.com

${ }^{1}$ Center for Epidemiological Research, Nykøbing Falster Hospital, Strandboulevarden 64, DK-4800 Nykøbing Falster, Denmark

Full list of author information is available at the end of the article
}

projected increase to 693 million by 2045 [2]. According to the International Diabetes Federation (IDF), more than 224 million adults with DM remain undiagnosed due to a combination of lack of awareness, poor health systems, slow onset of symptoms, and slow disease progression [2]. Undiagnosed, poorly or sub-controlled DM can cause lifelong complications, including kidney disease, heart disease, blindness and death, and undiagnosed DM is associated with a 1.5- to 3.0-fold mortality compared with no DM [3, 4]. A high-risk state for

(C) The Author(s). 2020 Open Access This article is licensed under a Creative Commons Attribution 4.0 International License, which permits use, sharing, adaptation, distribution and reproduction in any medium or format, as long as you give appropriate credit to the original author(s) and the source, provide a link to the Creative Commons licence, and indicate if changes were made. The images or other third party material in this article are included in the article's Creative Commons licence, unless indicated otherwise in a credit line to the material. If material is not included in the article's Creative Commons licence and your intended use is not permitted by statutory regulation or exceeds the permitted use, you will need to obtain permission directly from the copyright holder. To view a copy of this licence, visit http://creativecommons.org/licenses/by/4.0/. The Creative Commons Public Domain Dedication waiver (http://creativecommons.org/publicdomain/zero/1.0/) applies to the data made available in this article, unless otherwise stated in a credit line to the data. 
developing type $2 \mathrm{DM}$ (T2DM) is prediabetes; where the blood glucose level is above normal but below the diagnostic level of DM.

Public health actions focusing on identification, treatment and follow-up are essential for preventing and reducing the human, social and economic consequences of DM. This requires reliable estimates of prevalence and risk groups, and it requires easily available information about how to identify risk groups.

In Denmark, the incidence and prevalence of diagnosed DM are monitored using nationwide registers [5]. Some estimates of prediabetes and undiagnosed DM have been derived from population-based surveys [6] however, to our knowledge, no study has focused on DM related conditions in rural areas. Our study aimed to provide data from Lolland-Falster, a disadvantaged rural-provincial part of Denmark with a life expectancy below the national average [7], and with health problems reported more frequently than in the rest of the country [8]. Using data from a population-based survey in Lolland-Falster [9], we focused on prevalence and risk factors of prediabetes, undiagnosed DM, poorly and potentially sub-controlled DM. Though clinically distinguishable, these conditions share the need for intervention in terms of initiation and/or regulation of treatment.

\section{Methods}

\section{Study population}

Data were derived from the rural-Danish Lolland-Falster Health Study (LOFUS), a prospective cohort study that aims to establish baseline information on the health status of the inhabitants of Lolland-Falster [9]. In this study, persons aged 18 and above were randomly selected from the Danish Civil Registration System and invited with their household members of all ages to participate. Invitations were sent to all persons of the invited households either by electronic or ordinary mail. Invited persons who agreed to participate completed web-based questionnaires prior to the physical examination at one of the stationary sites. Half-way through the data collection, the overall participation rate was found to be $34.1 \%$ (men: $31.9 \%$, women: $36.4 \%$ ). A detailed description of the study protocol [9] and information on the socio-economic determinants of participation [10] have been published previously. For this study, we included adults aged 20 years and above $(n=10,895)$ recruited from 2016 to 2019, excluding women reported to be pregnant.

\section{Self-reported and health examination data}

From questionnaires, we used data on current selfperceived health, which was measured by one question with five response options ranging from excellent to very poor. One-item measures of self-rated general health are widely used in population studies and have shown independent associations with a variety of health outcomes including morbidity and mortality [11]. Leisure time physical activity was measured by one question where the respondents stated their level of physical activity in leisure time during the last 12 months in one of four response categories: (1) mainly sedentary activities (TVwatching, reading), (2) light physical activities $\geqq 4 \mathrm{~h}$ per week (walking, bicycling, light gardening), (3) sports or other more vigorous activities $\geq 4 \mathrm{~h}$ per week (heavy gardening), (4) highly vigorous physical activity several times per week (heavy exercise or competitive sports). Because of very few participants in the highest category of leisure time physical activity $n=268$, the variable was classified into: 'low'- (category 1), 'moderate'- (category 2), and 'high'- (category 3 and 4). Information on dietary habits was obtained from responses to one question regarding self-assessment of general dietary habits.

Furthermore, we used data on self-reported diabetes, insulin medication, medication with other antidiabetic drugs, cardiovascular disease (stroke, atherosclerosis, angina pectoris, deep-vein thrombosis, hypertension) and medication (antihypertensive medication, heart medication, diuretics, cholesterol lowering medications, anticoagulants), alcohol consumption, and smoking habits. Two-thirds of LOFUS participants reported rare or no alcohol consumption, a percentage not compatible with sales statistics [12], and data were missing for $10 \%$. We did therefore not use this information. From the health examination, we used data on measured height and weight.

\section{Blood and urine samples}

Non-fasting blood samples were collected at three study centers, and analyzed at the Department of Clinical Biochemistry at Nykøbing Falster Hospital accredited by the standard ISO 15189. We used data on glycated hemoglobin (HbA1c), triglycerides, serum high-density lipoprotein cholesterol (HDL) and serum total cholesterol. Furthermore, we used data on urine albumin-tocreatinine ratio (UACR) from spot urine tests.

\section{Diabetes definition}

No DM was defined as HbA1c levels below $42 \mathrm{mmol} /$ mol and no self-reported diagnosis of DM or use of antidiabetic medication including insulin and other antidiabetic medications. Prediabetes was defined, according to Danish guidelines, as HbA1c levels in the range of 42 $\mathrm{mmol} / \mathrm{mol}$ to $47 \mathrm{mmol} / \mathrm{mol}$ [13] and, no self-reported diagnosis of DM and/or use of antidiabetic medication.

We defined undiagnosed DM as HbA1c $\geqq 48$ and no self-reported diagnosis of DM and/or use of antidiabetic drugs, while diagnosed DM or DM-awareness was 
defined as reporting a DM diagnosis and/or use of antidiabetic medication. It was not possible to distinguish between type $1 \mathrm{DM}$ (T1DM) and T2DM due to lack of information.

We explored glycaemic control measured by HbA1c in participants who reported having a DM diagnosis and/or use of antidiabetic medication by 1) using the American Diabetes Association (ADA) recommendation on a reasonable HbA1c-level for adults of $<53 \mathrm{mmol} / \mathrm{mol}$ [14], and by 2) dividing the diabetic population into three groups: poorly controlled DM (HbA1c $\geqq 60 \mathrm{mmol} / \mathrm{mol}$ ); potentially sub-controlled DM (HbA1c $48-59 \mathrm{mmol} /$ $\mathrm{mol}$ ); and well-controlled DM (HbA1c $<48 \mathrm{mmol} / \mathrm{mol}$ ). Participants with missing data on HbA1c, self-reported diagnosis of DM and/ self-reported use of anti-diabetic medication were labeled "Missing/ unclassified".

We merged persons with prediabetes, undiagnosed DM, poorly controlled DM, and potentially subcontrolled DM into one group designated DM-related conditions in need of intervention.

\section{Body mass index}

Body mass index (BMI) was defined as weight in kilograms divided by height in meters squared $\left(\mathrm{kg} / \mathrm{m}^{2}\right)$, and divided into underweight (BMI less than 18.5) normal (BMI 18.5-24.9), overweight (BMI 25.0-29.9), and obese (BMI 30.0 or greater) [15].

\section{Statistical methods}

We tabulated the distribution of study participants by DM-related diagnostic groups. Based on the risk factors known from the survey, we calculated relative risks (RR), by unconditional maximum likelihood estimation (MLE), including 95\% confidence intervals (CI), using normal approximation, for presence of DM-related conditions in need of intervention. For risks found to be strongly associated with these conditions, we calculated also sensitivity and specificity, and corresponding 95\% confidence intervals using exact binomial test, of a hypothesized intervention. Simultaneous confidence intervals for multinomial proportions were calculated with the method of Sison and Glaz (see MultinomialCI package in $\mathrm{R}$ and refs. therein). Adjusted RR was obtained with a log-binomial model, and the $\mathrm{R}$ package 'logbin' was used.

Data handling, statistical analyses and plots were performed in $\mathrm{R}$ ver. 3.5.2, using dplyr, epitools, MultinomialCI, VennDiagram and RColorBrewer packages.

\section{Results}

Among the 10,895 LOFUS-participants aged 20 years and above, the prevalence of prediabetes was 5.8\% (95\% CI: 5.1-6.5). The overall DM prevalence was 6.1\% (95\% CI: 5.6-6.5) with a diagnosed to undiagnosed DM ratio of 5.3:0.8. The prevalence of undiagnosed DM was $0.8 \%$ (95\% CI: $0.1-1.4)$; poorly controlled DM $1.2 \%$ (95\% CI: $0.5-1.9$ ); potentially sub-controlled DM $2.0 \%$ (95\% CI: 1.4-2.7); and well-controlled DM 2.1\% (95\%CI: 1.4-2.8) (Table 1). In total, 9.8\% of the participants had DMrelated conditions in need of intervention. No DM was found in $81.7 \%$ (95\% CI: 81.0-82.4) and more frequent in women, $84.3 \%$, than in men, $79.0 \%$ (Supplementary Table 1). DM diagnosis was unclassified in $6.4 \%$ due to missing values for one or more DM defining factors (Supplementary Figure 1).

Among those diagnosed with DM, 42.8\% had HbA1c $\geq 53 \mathrm{mmol} / \mathrm{mol}$; a percentage slightly higher for men than for women. In persons diagnosed with DM, 22.1\% had poorly controlled DM and 38.5\% had potentially sub-controlled DM; percentages increasing with age (Supplementary Table 2).

Mean age was 64.8 years $(\mathrm{SD}=10.7)$ in the prediabetes group, 62.4 years $(\mathrm{SD}=10.1)$ in the undiagnosed $\mathrm{DM}$ group, 62.7 years $(\mathrm{SD}=11.7)$ in the poorly controlled $\mathrm{DM}$ group, and 66.3 years $(\mathrm{SD}=9.3)$ in the potentially sub-controlled DM group. With regard to lipid profiles, mean HDL concentration was $1.3 \pm 0.4 \mathrm{mmol} / \mathrm{l}$ in both prediabetes and undiagnosed DM groups, $1.2 \pm 0.4$ $\mathrm{mmol} / \mathrm{l}$ in the poorly controlled DM group, and $1.3 \pm$ $0.4 \mathrm{mmol} / \mathrm{l}$ in the potentially sub-controlled DM group. Mean total cholesterol concentration was $5.2 \pm 1.2$ $\mathrm{mmol} / \mathrm{l}$ in persons with prediabetes; $4.3 \pm 1.1 \mathrm{mmol} / \mathrm{l}$ in persons with undiagnosed DM and poorly controlled $\mathrm{DM}$, and $4.2 \pm 0.9 \mathrm{mmol} / \mathrm{l}$ in persons with potentially sub-controlled DM. Mean triglyceride concentration was $2.3 \pm 1.4 \mathrm{mmol} / \mathrm{l}$ among individuals with prediabetes; $2.2 \pm 1.4 \mathrm{mmol} / \mathrm{l}$ among individuals with undiagnosed $\mathrm{DM}, \quad 2.6 \pm 2.1 \mathrm{mmol} / \mathrm{l}$ among individuals with poorly controlled DM, and $2.2 \pm 1.2 \mathrm{mmol} / \mathrm{l}$ in individuals with potentially sub-controlled DM (Supplementary Table 3). Definitions of diagnostic groups can be found in Supplementary Table 4.

Men were at a higher risk than women of having a DM-related condition in need of intervention; RR 1.41 (95\% CI 1.26-1.58) (Table 2). This RR was almost identical when calculated based on only participants with diagnostic information; RR 1.42 (95\% CI 1.27-1.60), reflecting that persons with missing diagnostic information were almost randomly distributed between men and women. In the following, we focus on RRs calculated based only on persons with diagnostic information. Persons aged 60 years and above had a more than doubled risk compared with younger persons; RR 2.66 (95\% CI 2.34-3.01). Current and former smoking were associated with slightly increased risks as compared with never smoking; RR of 1.38 (95\% CI 1.19-1.62) and 1.45 (95\% CI 1.27-1.65), respectively. Self-reported health as being undecided compared with good was associated with an 
Table 1 Distribution by diabetes-related diagnostic groups of participants in Lolland-Falster Health Study aged 20 + years: Determinants, 2016-2019

\begin{tabular}{|c|c|c|c|c|c|c|}
\hline \multirow[t]{2}{*}{ Diagnostic group } & \multicolumn{2}{|l|}{ Men } & \multicolumn{2}{|c|}{ Women } & \multicolumn{2}{|l|}{ Total } \\
\hline & $n$ & $\%$ with $(95 \% \mathrm{Cl})$ & $\mathrm{n}$ & $\%$ with $(95 \% \mathrm{Cl})$ & $n$ & $\%$ with $(95 \% \mathrm{Cl})$ \\
\hline No DM & 4182 & $79(78.0-80.1)$ & 4722 & $84.3(83.4-85.2)$ & 8904 & $81.7(81.0-82.4)$ \\
\hline Prediabetes & 325 & $6.1(5.1-7.2)$ & 308 & $5.5(4.6-6.4)$ & 633 & $5.8(5.1-6.5)$ \\
\hline Undiagnosed DM & 55 & $1.0(0-2.1)$ & 27 & $0.5(0-1.4)$ & 82 & $0.8(0.1-1.4)$ \\
\hline Poorly controlled DM & 81 & $1.5(0.5-2.6)$ & 47 & $0.8(0-1.7)$ & 128 & $1.2(0.5-1.9)$ \\
\hline Potentially sub-controlled DM & 148 & $2.8(1.8-3.9)$ & 75 & $1.3(0.4-2.2)$ & 223 & $2.0(1.4-2.7)$ \\
\hline Well-controlled DM & 138 & $2.6(1.6-3.7)$ & 90 & $1.6(0.7-2.5)$ & 228 & $2.1(1.4-2.8)$ \\
\hline Missing/ unclassified & 362 & $6.8(5.8-7.9)$ & 335 & $6.0(5.1-6.9)$ & 697 & $6.4(5.7-7.1)$ \\
\hline Total with missing/ unclassified & 5291 & 100 & 5604 & 100 & 10,895 & 100 \\
\hline Total excluding missing/ unclassified & 4929 & 93.2 & 5269 & 94.0 & 10,198 & 93.6 \\
\hline DMRC & 609 & $11.5(10.7-12.4)$ & 457 & $8.2(7.5-8.9)$ & 1066 & $9.8(9.2-10.4)$ \\
\hline DM diagnosed & 367 & 100 & 212 & 100 & 579 & 100 \\
\hline - $\mathrm{HbA} 1 \mathrm{c} \geq 53 \mathrm{mmol} / \mathrm{mol}$ & 160 & $43.6(38.4-48.9)$ & 88 & $41.5(34.9-48.4)$ & 248 & $42.8(38.7-47.0)$ \\
\hline$-\mathrm{HbA} 1 \mathrm{c}<53 \mathrm{mmol} / \mathrm{mol}$ & 207 & $56.4(51.2-61.7)$ & 124 & $58.5(51.9-65.4)$ & 331 & $57.2(53.0-61.4)$ \\
\hline
\end{tabular}

DMRC diabetes-related conditions in need of intervention

increased risk; RR 1.88 (95\% CI 1.67-2.12); and so was poor self-reported health; RR 2.59 (95\% CI 2.13-3.15). Presence of self-reported hypertension as compared with absence was associated with a RR of 3.28 (95\% CI 2.933.68). Low and moderate leisure time physical activity as being compared with high leisure time physical activity; RR of 2.64 (95\% CI 2.17-3.22) and 1.84(1.56-2.17). Overweight as compared with normal weight was associated with an increased risk; RR 2.40 (95\% CI 2.0-2.87); and so was obesity; RR 4.51 (95\% CI 3.79-5.38). The RR for DM-related conditions in obese persons adjusted for health perception, smoking, hypertension, physical activity, and dietary was 3.28 (2.73-3.93).

Unhealthy dietary habits were not found to be associated with higher risk when compared with healthy/ very healthy; RR of 0.98 (95\% CI 0.74-1.29).

The results in Table 2 can form the basis for easy identification in health care and/or social service institutions of persons with DM-related conditions in need of intervention. As $10 \%$ of all adult LOFUS-participants had a DM-related condition in need of intervention, we focused on the risk factors showing a doubling of the prevalence proportion. Namely, among persons with poor self-perceived health those with DM-related conditions constituted 21.2\%; among persons with selfreported hypertension 20.7\%; among persons reporting low leisure time physical activity 16.1\%; and among obese persons 19.6\% (Table 2).

However, for potential interventions both the sensitivity and the specificity need to be known. Interventions targeting persons with poor self-perceived health would identify only $9.0 \%$ (95\% CI 7.4-10.9) of persons with
DM-related conditions in need of intervention, but it would on the other hand cause limited harm as it would target very few persons without the conditions; the specificity being 96.1\% (95\% CI 95.7-96.5) (Table 3).

Interventions targeting persons with low leisure time physical activity would identify $18.3 \%$ (95\% CI 16.2-21.0) of persons with DM-related conditions in need of intervention, with a specificity of 88.8 (CI 88.1-89.4). Intervention towards obese persons or persons with hypertension would identify between 46.6\% (95\% CI 43.5-49.6) and 57.7\% (95\% CI 54.660.7 ) of the persons with the DM-related conditions in need of intervention, and with specificities between 74.3\% (95\% CI 73.4-75.2) and 77.7\% (95\% CI 76.878.5) only about one fourth of targeted persons would be without the conditions.

For comparison, we calculated also sensitivity and specificity for an intervention towards all persons aged 60 years and above. An intervention towards this group would be effective, as $70.5 \%$ (95\% CI 67.7-73.3) of those with the DM-related conditions would be identified, but with a specificity of only $55.3 \%$ (95\% CI 54.3-56.3) almost half of the targeted persons would not have the conditions.

Cardiovascular disease was reported in $55.3 \%$ of adults with a DM-related condition in need of intervention, and cardiovascular medication in 68.9\%; 24.7 and $29.7 \%$ in adults with no DM; 72.0 and $85.1 \%$ in persons with well-controlled DM. Micro- and macro-albuminuria were observed more frequently in persons with DMrelated conditions in need of intervention, 22.1 and $4.0 \%$, respectively, than in persons with no DM, 11.6 and $0.7 \%$, respectively (Table 4 ). 
Table 2 Relative Risk of diabetes-related conditions in need of intervention

\begin{tabular}{|c|c|c|c|c|c|c|c|c|}
\hline & \multirow{2}{*}{$\begin{array}{l}\text { Total } \\
\text { n (\%) }\end{array}$} & \multicolumn{4}{|c|}{ Diagnostic group } & \multirow{2}{*}{$\begin{array}{l}\text { RR of } \\
\text { DMRC } \\
\text { (total) }\end{array}$} & \multirow{2}{*}{$\begin{array}{l}\text { RR of } \\
\text { DMRC excl. } \\
\text { Missing/ } \\
\text { unclassified }\end{array}$} & \multirow{2}{*}{$\begin{array}{l}\text { DMRC \% } \\
\text { excl. } \\
\text { Missing/ } \\
\text { unclassified }\end{array}$} \\
\hline & & $\begin{array}{l}\text { No } \\
\text { DM } \\
\mathrm{n} \\
(\%) \\
\end{array}$ & $\begin{array}{l}\text { DMRC } \\
\text { n (\%) }\end{array}$ & $\begin{array}{l}\text { WCDM } \\
\text { n (\%) }\end{array}$ & $\begin{array}{l}\text { Missing/ } \\
\text { unclassified } \\
\text { n (\%) }\end{array}$ & & & \\
\hline$\overline{\text { Total }}$ & $\begin{array}{l}10 \\
895 \\
(100)\end{array}$ & $\begin{array}{l}8904 \\
(81.7)\end{array}$ & $\begin{array}{l}1066 \\
(9.8)\end{array}$ & $\begin{array}{l}228 \\
(2.1)\end{array}$ & $697(6.4)$ & & & 10.5 \\
\hline Men & $\begin{array}{l}5291 \\
(48.6)\end{array}$ & $\begin{array}{l}4182 \\
(79.0)\end{array}$ & $\begin{array}{l}609 \\
(11.5)\end{array}$ & $\begin{array}{l}138 \\
(2.6)\end{array}$ & $362(6.8)$ & $\begin{array}{l}1.41(1.26- \\
1.58)\end{array}$ & $\begin{array}{l}1.42(1.27- \\
1.60)\end{array}$ & 12.4 \\
\hline Women & $\begin{array}{l}5604 \\
(51.4)\end{array}$ & $\begin{array}{l}4722 \\
(84.3)\end{array}$ & $\begin{array}{l}457 \\
(8.2)\end{array}$ & $90(1.6)$ & $335(6.0)$ & 1 & 1 & 8.7 \\
\hline \multicolumn{9}{|l|}{ Age } \\
\hline$-20-59$ & $\begin{array}{l}5789 \\
(53.1)\end{array}$ & $\begin{array}{l}5014 \\
(86.6)\end{array}$ & $\begin{array}{l}314 \\
(5.4)\end{array}$ & $36(0.6)$ & $425(7.3)$ & 1 & 1 & 5.9 \\
\hline$-60+$ & $\begin{array}{l}5106 \\
(46.9)\end{array}$ & $\begin{array}{l}3890 \\
(76.2)\end{array}$ & $\begin{array}{l}752 \\
(14.7)\end{array}$ & $\begin{array}{l}192 \\
(3.8)\end{array}$ & $272(5.3)$ & $\begin{array}{l}2.71(2.39- \\
3.08)\end{array}$ & $\begin{array}{l}2.66(2.34- \\
3.01)\end{array}$ & 15.6 \\
\hline \multicolumn{9}{|l|}{ Smoking } \\
\hline - Current & $\begin{array}{l}1997 \\
(18.3)\end{array}$ & $\begin{array}{l}1680 \\
(84.1)\end{array}$ & $\begin{array}{l}228 \\
(11.4)\end{array}$ & $38(1.9)$ & $51(2.6)$ & $\begin{array}{l}1.38(1.19- \\
1.62)\end{array}$ & $\begin{array}{l}1.39(1.19- \\
1.63)\end{array}$ & 11.7 \\
\hline - Former & $\begin{array}{l}3721 \\
(34.2)\end{array}$ & $\begin{array}{l}3084 \\
(82.9)\end{array}$ & $\begin{array}{l}445 \\
(12.0)\end{array}$ & $\begin{array}{l}118 \\
(3.2)\end{array}$ & $74(2.0)$ & $\begin{array}{l}1.45(1.27- \\
1.65)\end{array}$ & $\begin{array}{l}1.45(1.28- \\
1.65)\end{array}$ & 12.2 \\
\hline - Never & $\begin{array}{l}4658 \\
(42.8)\end{array}$ & $\begin{array}{l}4116 \\
(88.4)\end{array}$ & $\begin{array}{l}384 \\
(8.2)\end{array}$ & $71(1.5)$ & $87(1.9)$ & 1 & 1 & 8.4 \\
\hline - Missing & $\begin{array}{l}519 \\
(4.8)\end{array}$ & $\begin{array}{l}24 \\
(4.6)\end{array}$ & $9(1.7)$ & $1(0.2)$ & $485(93.5)$ & $\begin{array}{l}0.21(0.11- \\
0.40)\end{array}$ & $\begin{array}{l}3.15(1.78- \\
5.56)\end{array}$ & 26.5 \\
\hline \multicolumn{9}{|l|}{ Health status, self-reported } \\
\hline - Good/very good & $\begin{array}{l}7154 \\
(65.7)\end{array}$ & $\begin{array}{l}6366 \\
(89.0)\end{array}$ & $\begin{array}{l}559 \\
(7.8)\end{array}$ & $\begin{array}{l}112 \\
(1.6)\end{array}$ & $117(1.6)$ & 1 & 1 & 7.9 \\
\hline - Neither good/nor bad & $\begin{array}{l}2770 \\
(25.4)\end{array}$ & $\begin{array}{l}2178 \\
(78.6)\end{array}$ & $\begin{array}{l}407 \\
(14.7)\end{array}$ & $\begin{array}{l}100 \\
(3.6)\end{array}$ & $85(3.1)$ & $\begin{array}{l}1.88(1.67- \\
2.12)\end{array}$ & $\begin{array}{l}1.91(1.69- \\
2.15)\end{array}$ & 15.2 \\
\hline - Poor/very poor & $\begin{array}{l}474 \\
(4.4)\end{array}$ & $\begin{array}{l}342 \\
(72.2)\end{array}$ & $\begin{array}{l}96 \\
(20.3)\end{array}$ & $16(3.4)$ & $20(4.2)$ & $\begin{array}{l}2.59(2.13- \\
3.15)\end{array}$ & $\begin{array}{l}2.66(2.19- \\
3.23)\end{array}$ & 21.2 \\
\hline -Missing/unclassified & $\begin{array}{l}497 \\
(4.6)\end{array}$ & $\begin{array}{l}18 \\
(3.6)\end{array}$ & $4(0.8)$ & 0 & 475 (95.6) & $\begin{array}{l}0.10(0.04- \\
0.27)\end{array}$ & $\begin{array}{l}2.29(0.94- \\
5.57)\end{array}$ & 18.2 \\
\hline \multicolumn{9}{|l|}{ Hypertension, self-reported } \\
\hline - Yes & $\begin{array}{l}3004 \\
(27.6)\end{array}$ & $\begin{array}{l}2156 \\
(71.8)\end{array}$ & $\begin{array}{l}606 \\
(20.2)\end{array}$ & $\begin{array}{l}164 \\
(5.5)\end{array}$ & $78(2.6)$ & $\begin{array}{l}3.28(2.93- \\
3.68)\end{array}$ & $\begin{array}{l}3.33(2.97- \\
3.73)\end{array}$ & 20.7 \\
\hline$-\mathrm{No}$ & $\begin{array}{l}7244 \\
(66.5)\end{array}$ & $\begin{array}{l}6642 \\
(91.7)\end{array}$ & $\begin{array}{l}445 \\
(6.1)\end{array}$ & $61(0.8)$ & $96(1.3)$ & 1 & 1 & 93.0 \\
\hline - Missing/unclassified & $\begin{array}{l}647 \\
(5.9)\end{array}$ & $\begin{array}{l}106 \\
(16.4)\end{array}$ & $\begin{array}{l}15 \\
(2.3)\end{array}$ & $3(0.5)$ & $523(80.8)$ & $\begin{array}{l}0.38(0.23- \\
0.63)\end{array}$ & $\begin{array}{l}1.94(1.20- \\
3.15)\end{array}$ & 12.1 \\
\hline \multicolumn{9}{|l|}{ Dietary } \\
\hline - Very healthy/healthy & $\begin{array}{l}4946 \\
(45.4)\end{array}$ & $\begin{array}{l}4268 \\
(86.3)\end{array}$ & $\begin{array}{l}473 \\
(9.6)\end{array}$ & $\begin{array}{l}111 \\
(2.2)\end{array}$ & $94(1.9)$ & 1 & 1 & 9.8 \\
\hline - Roughly healthy & $\begin{array}{l}4901 \\
(45.0)\end{array}$ & $\begin{array}{l}4155 \\
(84.8)\end{array}$ & $\begin{array}{l}537 \\
(11.0)\end{array}$ & $\begin{array}{l}107 \\
(2.2)\end{array}$ & $102(2.1)$ & $\begin{array}{l}1.15(1.02- \\
1.29)\end{array}$ & $\begin{array}{l}1.15(1.02- \\
1.29)\end{array}$ & 11.2 \\
\hline - Unhealhty/very unhealthy & $\begin{array}{l}534 \\
(4.9)\end{array}$ & $\begin{array}{l}456 \\
(85.4)\end{array}$ & $\begin{array}{l}50 \\
(9.4)\end{array}$ & $9(1.7)$ & 19 (3.6) & $\begin{array}{l}0.98(0.74- \\
1.29)\end{array}$ & $1(0.75-1.31)$ & 9.7 \\
\hline - Missing/unclassified & $\begin{array}{l}514 \\
(4.7)\end{array}$ & $\begin{array}{l}25 \\
(4.9)\end{array}$ & $6(1.2)$ & $1(0.2)$ & $482(93.8)$ & $\begin{array}{l}0.12(0.05- \\
0.27)\end{array}$ & $\begin{array}{l}1.92(0.93- \\
3.98)\end{array}$ & 20.0 \\
\hline \multicolumn{9}{|l|}{ Physical activity } \\
\hline - Low (mainly sedentary) & $\begin{array}{l}1245 \\
(11.4)\end{array}$ & $\begin{array}{l}969 \\
(77.8)\end{array}$ & $\begin{array}{l}195 \\
(15.7)\end{array}$ & $47(3.8)$ & $34(2.7)$ & $\begin{array}{l}2.64(2.17- \\
3.22)\end{array}$ & $\begin{array}{l}2.67(2.2- \\
3.26)\end{array}$ & 16.1 \\
\hline
\end{tabular}


Table 2 Relative Risk of diabetes-related conditions in need of intervention (Continued)

\begin{tabular}{|c|c|c|c|c|c|c|c|c|}
\hline & \multirow{2}{*}{$\begin{array}{l}\text { Total } \\
\text { n (\%) }\end{array}$} & \multicolumn{4}{|c|}{ Diagnostic group } & \multirow{2}{*}{$\begin{array}{l}\text { RR of } \\
\text { DMRC } \\
\text { (total) }\end{array}$} & \multirow{2}{*}{$\begin{array}{l}\text { RR of } \\
\text { DMRC excl. } \\
\text { Missing// } \\
\text { unclassified }\end{array}$} & \multirow{2}{*}{$\begin{array}{l}\text { DMRC \% } \\
\text { excl. } \\
\text { Missing/ } \\
\text { unclassified }\end{array}$} \\
\hline & & $\begin{array}{l}\text { No } \\
\text { DM } \\
\text { n } \\
(\%)\end{array}$ & $\begin{array}{l}\text { DMRC } \\
\mathrm{n}(\%)\end{array}$ & $\begin{array}{l}\text { WCDM } \\
\text { n (\%) }\end{array}$ & $\begin{array}{l}\text { Missing/ } \\
\text { unclassified } \\
\text { n (\%) }\end{array}$ & & & \\
\hline - Moderate (light physical activities $\geqq 4 \mathrm{~h}$ per week) & $\begin{array}{l}6356 \\
(58.3)\end{array}$ & $\begin{array}{l}5395 \\
(84.9)\end{array}$ & $\begin{array}{l}694 \\
(10.9)\end{array}$ & $\begin{array}{l}134 \\
(2.1)\end{array}$ & $133(2.1)$ & $\begin{array}{l}1.84(1.56- \\
2.17)\end{array}$ & $\begin{array}{l}1.85(1.57- \\
2.18)\end{array}$ & 11.2 \\
\hline $\begin{array}{l}\text { - High (sports or other more vigorous activities } \geq 4 \mathrm{~h} \text { per } \\
\text { week/highly vigorous physical activity several times per } \\
\text { week) }\end{array}$ & $\begin{array}{l}2732 \\
(25.1)\end{array}$ & $\begin{array}{l}2484 \\
(91.0)\end{array}$ & $\begin{array}{l}162 \\
(5.9)\end{array}$ & $44(1.6)$ & $42(1.5)$ & 1 & 1 & 6.0 \\
\hline - Missing & $\begin{array}{l}562 \\
(5.2)\end{array}$ & $\begin{array}{l}56 \\
(10.0)\end{array}$ & $\begin{array}{l}15 \\
(2.7)\end{array}$ & $3(0.5)$ & $488(86.8)$ & $\begin{array}{l}0.45(0.27- \\
0.76)\end{array}$ & $\begin{array}{l}3.37(2.09- \\
5.42)\end{array}$ & 20.3 \\
\hline \multicolumn{9}{|l|}{ BMI } \\
\hline - Underweight & $\begin{array}{l}124 \\
(1.1)\end{array}$ & $\begin{array}{l}111 \\
(89.5)\end{array}$ & $4(3.2)$ & 0 & $9(7.3)$ & $\begin{array}{l}0.80(0.30- \\
2.12)\end{array}$ & $\begin{array}{l}0.81(0.31- \\
2.16)\end{array}$ & 3.5 \\
\hline - Normal & $\begin{array}{l}3743 \\
(34.4)\end{array}$ & $\begin{array}{l}3349 \\
(89.5)\end{array}$ & $\begin{array}{l}151 \\
(4.0)\end{array}$ & $36(1.0)$ & $207(5.5)$ & 1 & 1 & 4.3 \\
\hline - Overweight & $\begin{array}{l}4260 \\
(39.1)\end{array}$ & $\begin{array}{l}3470 \\
(81.5)\end{array}$ & $\begin{array}{l}412 \\
(9.7)\end{array}$ & $95(2.2)$ & $283(6.6)$ & $\begin{array}{l}2.40(2.0- \\
2.87)\end{array}$ & $\begin{array}{l}2.43(2.02- \\
2.91)\end{array}$ & 10.4 \\
\hline - Obese & $\begin{array}{l}2712 \\
(24.9)\end{array}$ & $\begin{array}{l}1932 \\
(71.2)\end{array}$ & $\begin{array}{l}494 \\
(18.2)\end{array}$ & $95(3.5)$ & $191(7.0)$ & $\begin{array}{l}4.51(3.79- \\
5.38)\end{array}$ & $\begin{array}{l}4.59(3.85- \\
5.46)\end{array}$ & 19.6 \\
\hline - Missing/unclassified & $\begin{array}{l}56 \\
(0.5)\end{array}$ & $\begin{array}{l}42 \\
(75.0)\end{array}$ & $5(9.0)$ & $2(3.6)$ & $7(12.5)$ & $\begin{array}{l}2.21(0.94- \\
5.18)\end{array}$ & $\begin{array}{l}2.39(1.03- \\
5.56)\end{array}$ & 10.6 \\
\hline \multicolumn{9}{|l|}{ Lipids (mean $\pm \mathrm{SD}$ ) mmol/L } \\
\hline $\mathrm{HDL}$ & $\begin{array}{l}1.5 \pm \\
0.4\end{array}$ & $\begin{array}{l}1.5 \pm \\
0.4\end{array}$ & $\begin{array}{l}1.3 \pm \\
0.4\end{array}$ & $\begin{array}{l}1.3 \pm \\
0.4\end{array}$ & $1.4 \pm 0.4$ & & & \\
\hline Total cholesterol & $\begin{array}{l}5.1 \pm \\
1.1\end{array}$ & $\begin{array}{l}5.2 \pm \\
1.1\end{array}$ & $\begin{array}{l}4.9 \pm \\
1.2\end{array}$ & $\begin{array}{l}4.4 \pm \\
1.1\end{array}$ & $5.0 \pm 1.1$ & & & \\
\hline Triglycerides & $\begin{array}{l}1.8 \pm \\
1.2\end{array}$ & $\begin{array}{l}1.7 \pm \\
1.1\end{array}$ & $\begin{array}{l}2.4 \pm \\
1.5\end{array}$ & $\begin{array}{l}2.0 \pm \\
1.0\end{array}$ & $1.9 \pm 1.3$ & & & \\
\hline
\end{tabular}

DMRC diabetes-related conditions in need of intervention, WCDM well-controlled diabetes

\section{Discussion}

\section{Main findings}

One in 10 participants in the rural-provincial LollandFalster Health Study had a DM-related condition that required further investigation and/or better glycaemic control. As these conditions may lead to serious health consequences, the study revealed a considerable unmet need for prevention. The risk of undiagnosed and/or poorly controlled conditions was more than doubled among persons with self-reported poor health status;

Table 3 Sensitivity and specificity of detecting diabetes-related conditions in need of intervention

\begin{tabular}{|c|c|c|c|c|c|c|c|c|}
\hline \multirow[t]{2}{*}{ Exposure } & \multicolumn{2}{|c|}{ Exposed } & \multicolumn{2}{|c|}{ Non-exposed } & \multirow{2}{*}{$\begin{array}{l}\text { Sensitivity } \\
(95 \% \mathrm{Cl})\end{array}$} & \multirow{2}{*}{$\begin{array}{l}\text { Specificity } \\
(95 \% \mathrm{Cl})\end{array}$} & \multirow{2}{*}{$\begin{array}{l}\text { Positive } \\
\text { predictive } \\
\text { value ( } 95 \% \\
\text { Cl) }\end{array}$} & \multirow{2}{*}{$\begin{array}{l}\text { Negative } \\
\text { predictive } \\
\text { value }(95 \% \\
\text { Cl) }\end{array}$} \\
\hline & $\begin{array}{l}\text { DMRC } \\
\mathrm{n}\end{array}$ & $\begin{array}{l}\text { Non- } \\
\text { DMRC } \\
n\end{array}$ & $\begin{array}{l}\text { DMRC } \\
n\end{array}$ & $\begin{array}{l}\text { Non- } \\
\text { DMRC } \\
n\end{array}$ & & & & \\
\hline Age 60+ & 752 & 4082 & 314 & 5050 & $\begin{array}{l}70.5 \%(67.7- \\
73.3)\end{array}$ & $\begin{array}{l}55.3 \%(54.3- \\
56.3)\end{array}$ & $\begin{array}{l}15.6 \%(14.5- \\
16.6)\end{array}$ & $\begin{array}{l}94.1 \%(93.5- \\
94.8)\end{array}$ \\
\hline $\begin{array}{l}\text { Poor/very poor health status, self- } \\
\text { reported }\end{array}$ & 96 & 358 & 966 & 8756 & $9.0 \%(7.4-10.9)$ & $\begin{array}{l}96.1 \%(95.7- \\
96.5)\end{array}$ & $\begin{array}{l}21.1 \%(17.5- \\
25.2)\end{array}$ & $\begin{array}{l}90.1 \%(89.5- \\
90.7)\end{array}$ \\
\hline Hypertension, self-reported & 606 & 2320 & 445 & 6703 & $\begin{array}{l}57.7 \%(54.6- \\
60.7)\end{array}$ & $\begin{array}{l}74.3 \%(73.4- \\
75.2)\end{array}$ & $\begin{array}{l}20.7 \%(19.3- \\
22.2)\end{array}$ & $\begin{array}{l}93.8 \%(93.2- \\
94.3)\end{array}$ \\
\hline Low physical activity & 195 & 1016 & 856 & 8057 & $\begin{array}{l}18.3 \%(16.2- \\
21.0)\end{array}$ & $\begin{array}{l}88.8 \%(88.1- \\
89.4)\end{array}$ & $\begin{array}{l}16.1 \%(14.1- \\
18.3)\end{array}$ & $\begin{array}{l}90.4 \%(89.8- \\
91.0)\end{array}$ \\
\hline Obesity, measured & 494 & 2027 & 567 & 7061 & $\begin{array}{l}46.6 \%(43.5- \\
49.6)\end{array}$ & $\begin{array}{l}77.7 \%(76.8- \\
78.5)\end{array}$ & $\begin{array}{l}19.6 \%(18.1- \\
21.2)\end{array}$ & $\begin{array}{l}92.6 \%(92.0- \\
93.1)\end{array}$ \\
\hline
\end{tabular}

DMRC diabetes-related conditions in need of intervention 
Table 4 Cardiovascular disease and albuminuria

\begin{tabular}{|c|c|c|c|c|c|}
\hline & $\begin{array}{l}\text { Total } \\
\text { n (\%) } \\
\end{array}$ & $\begin{array}{l}\text { No DM } \\
\mathrm{n}(\%)\end{array}$ & $\begin{array}{l}\text { DMRC } \\
\mathrm{n}(\%)\end{array}$ & $\begin{array}{l}\text { WCDM } \\
\mathrm{n}(\%)\end{array}$ & $\begin{array}{l}\text { Missing/ unclassified } \\
\mathrm{n}(\%)\end{array}$ \\
\hline Overall & $10,895(100)$ & $8904(81.7)$ & $1066(9.8)$ & $228(2.1)$ & $697(6.4)$ \\
\hline \multicolumn{6}{|c|}{ Cardiovascular medication self-reported $^{a}$} \\
\hline - Yes & $3666(33.7)$ & $2643(29.7)$ & $734(68.9)$ & $194(85.1)$ & 95 (13.6) \\
\hline - No & $6580(60.4)$ & $6145(69.0)$ & $317(29.7)$ & $33(14.5)$ & $85(12.2)$ \\
\hline - Missing/ unclassified & $649(6.0)$ & $116(1.3)$ & $15(1.4)$ & $1(0.4)$ & $517(74.2)$ \\
\hline \multicolumn{6}{|l|}{ Cardiovascular disease self-reported $^{a}$} \\
\hline - Yes & $3016(27.7)$ & $2197(24.7)$ & $589(55.3)$ & $164(72.0)$ & $66(9.5)$ \\
\hline- No & $7400(67.9)$ & $6707(75.3)$ & $477(44.7)$ & $64(28.1)$ & $152(21.8)$ \\
\hline - Missing/unclassified & $479(4.4)$ & 0 & 0 & 0 & $479(68.7)$ \\
\hline \multicolumn{6}{|c|}{ Urine albumin-to-creatinine ratio mg/g } \\
\hline$-0-29$ & $6106(56.0)$ & $5047(56.7)$ & $567(53.2)$ & $106(46.5)$ & $386(55.4)$ \\
\hline - 30-299 (micro albuminuria) & $1410(13.0)$ & $1034(11.6)$ & $236(22.1)$ & $51(22.4)$ & $89(12.8)$ \\
\hline - $\geq 300$ (macro albuminuria) & $130(1.2)$ & $66(0.7)$ & $43(4.0)$ & $12(5.3)$ & $9(1.3)$ \\
\hline - Missing/unclassified & 3249 (29.8) & $2757(31.0)$ & $220(20.6)$ & $59(25.9)$ & $213(30.6)$ \\
\hline
\end{tabular}

DMRC diabetes-related conditions in need of intervention, WCDM well-controlled diabetes

${ }^{a}$ Cardiovascular disease and medication includes hypertension diagnosis and antihypertensive medication

with self-reported hypertension; with low self-reported leisure time physical activities or with obesity. Within these risk groups, $20 \%$ had an undiagnosed and/or poorly controlled condition. On this basis, health care and social service workers could in general recommend persons with these characteristics to have a blood sample analyzed and to seek advice on treatment. Provided that these services are easily available, such a simple, targeted strategy could potentially save many persons from developing severe life-long complications of DM.

\section{Comparison with other studies}

The prevalence of prediabetes of $5.8 \%$ in the LOFUS population was slightly higher than the $4.3 \%$ found in the Danish Health Examination Survey (DANHES), but lower than the $6.9 \%$ in the Danish General Suburban Population Study (GESUS) [6].

The prevalence of undiagnosed and diagnosed DM was 0.8 and $5.3 \%$, respectively. The prevalence was higher in men than in women, and while the reasons for this remain poorly understood, biological and psychosocial factors may play a role $[16,17]$, and women visit primary care clinics and diagnostic services more frequently than men $[18,19]$. Our prevalence of undiagnosed DM of $0.8 \%$ was higher than the $0.3 \%$ in GESUS and the $0.2 \%$ in the urban Copenhagen General Population Study (CGPS) [20], but lower than the $1.4 \%$ in DANHES based on slightly different definitions and data sources [6].

Our findings on health related characteristics associated with prediabetes and DM were consistent with previous studies; low leisure time physical activity and obesity are well established risk factors for DM-related conditions $[21,22]$ and so is hypertension as DM and high blood pressure share a number of potential risk factors, and are likely to cluster together along with other cardio-metabolic conditions [23]. The association between poor self-reported health status and DM-related conditions collaborate findings from Italy of a high correlation between elevated $\mathrm{HbA1c}$ concentrations and poor self-reported health status [24], and from Sweden where adults with high-risk HbAlc concentrations reported poor health status and had low level of health related quality of life [25]. Unhealthy dietary habits are associated with metabolic changes and increased risk of DM-related conditions [26]. However, this was not found in our study and could be related to the less comprehensive questionnaire applied.

The risk of DM increases with age resulting from increasing insulin resistance and impaired pancreatic islet function [27]. Older adults with DM are also at substantial risk for both acute and chronic macrovascular and cardiovascular complications. Our findings on age as a risk factor is therefore in concordance with the literature [28].

Triglyceride concentrations were slightly higher in the prediabetes, undiagnosed DM and poorly controlled DM groups when compared with the no DM group, consistent with previous studies [28, 29]. Some previous studies have reported lower HDL levels and higher total cholesterol concentrations in persons with prediabetes and DM $[23,30]$, but this was not found in our population.

Adults with DM-related conditions in need of intervention reported more frequently having cardiovascular disease or using cardiovascular medication than adults 
with no DM. There is evidence that chronic high blood glucose levels are associated with a substantially higher risk of cardiovascular disease [31], and patients with cardiovascular disease are reported to have a high risk of developing DM [32]. Because of the known relationship between cardiovascular disease and DM, early detection of DM related conditions in need of intervention are essential in order to prevent and delay progressive conditions.

A substantial proportion of adults with a DM-related condition in need of intervention had evidence of microand macro-albuminuria; identification and follow up is necessary to prevent and delay chronic kidney damage in this group. Previous studies found it might be efficient to test kidney function in adults with prediabetes or in persons at risk of having DM in order to determine chronic kidney disease at an early stage to prevent further complications [33, 34].

In LOFUS, approximately $43 \%$ of the persons diagnosed with DM had HbA1c levels $\geqq 53 \mathrm{mmol} / \mathrm{mol}$. Similar percentages have been reported in other countries globally $[35,36]$. In the U.S. National Health and Nutrition Examination Survey (NHANES) from 2007 to 2010, $47.5 \%$ of adults with DM had HbA1c levels $\geq 53$ [35]. In a Korean survey, $50.9 \%$ of adults with DM had HbA1c $\geq 53$ in 2010 [36]. Although these studies differed in population, sample sizes, and survey period, the results showed that glycemic control is challenged around the globe.

\section{Limitations}

This cross-sectional study had a number of limitations. First, only $34.1 \%$ of persons invited participated in LOFUS, which may lead to selection bias. There was an overall higher participation rate among individuals of higher income, education level, employment status, middle-aged (aged 50-60 years) and married [10]. It is already known that survey participation is lower among people of poorer socio-economic status and with disease compared with those without [37]. This can result in underestimation of the prevalence of prediabetes, undiagnosed DM, and poorly controlled DM, if the survey prevalence is taken at face value. Thus, our results likely represented conservative estimates of the true unmet need in a deprived area of Denmark. Second, while we were not able to distinguish between T1DM and T2DM, we assumed that only a small number of participants had T1DM. Third, our definitions of undiagnosed DM, prediabetes and poorly controlled DM may cause underestimation of conditions due to missing data, see Supplementary Figure 1. Fourth, data on alcohol consumption were considered unreliable and not used in the analysis. Moreover, oral glucose tolerance test (OGTT) was not performed in LOFUS and therefore only $\mathrm{HbA} 1 \mathrm{c}$ measurements were used for the analyses. Finally, differences in methods for the analysis of HbA1c across surveys influenced the comparison of estimates; however, it is not possible to determine the direction of such potential bias.

\section{Clinical implications}

Long-term care of DM patients is difficult and presents a considerable challenge to health care systems [38]. In order to evaluate DM management, it has been widely agreed that glycaemic control along with lipid profiles and blood pressure is the key in preventing complications. According to the American Diabetes Association, a reasonable target $\mathrm{HbA1c}$ value for many non-pregnant adults DM patients is $53 \mathrm{mmol} / \mathrm{mol}$ [12]. However, it has been suggested that an individualized therapeutic approach rather than a 'one-size-fits-all' strategy is essential to ensuring maximum success [14, 38].

Several DM risk scoring tools, including the Danish Diabetes Risk Score and the Leicester Risk Assessment Score, are available in order to enhance detection of undiagnosed DM $[39,40]$. These scores are based on simple anamnestic information and clinical observations and are recommended since uncertainty persists concerning benefits of population-based screening for DM [41]. However, these tools are primarily applied by medical doctors and used for consultations regarding DM.

Our study indicates that a substantial part of persons with DM-related conditions that would require further diagnostics and/or improved glycaemic control could be identified based on self-reported poor health and/or hypertension, and/ or low leisure time physical activity, and/or presence of obesity. This information is easily available for health care and social service workers simply from asking the person and from visually evaluating their body constitution. On this basis, health care and social service workers could in general recommend citizens with these characteristics to have a blood sample analyzed and to seek advice on treatment. Provided that these services are easily available for the citizens, such a simple, targeted strategy could potentially save many persons from developing severe life-long complications of DM.

\section{Conclusion}

In the Lolland-Falster Health Study, about $10 \%$ of participants had prediabetes, undiagnosed DM, poorly controlled or potentially sub-controlled DM. The risk of these conditions was more than doubled in persons with poor self-perceived health, self-reported hypertension, low leisure time physical activity or obesity. These findings can form the basis for a targeted effort to improve the prevention of DM and its complications. 


\section{Supplementary Information}

The online version contains supplementary material available at https://doi. org/10.1186/s12889-020-09791-2

Additional file 1 : Supplementary Table 1. Prevalence of prediabetes, undiagnosed and diagnosed diabetes according to gender and age. Supplementary Table $\mathbf{2}$. Diagnosed diabetes disaggregated by glycemic control according to gender and age. Supplementary Table 3. Descriptive characteristics of LOFUS participants by glycemic status. Supplementary Table 4. Definitions of diagnostic groups. Supplementary Figure 1. Venn diagram for missing/ unclassified data for one or more diabetes defining factors.

\section{Abbreviations}

DM: Diabetes mellitus; IDF: The International Diabetes Federation; T1DM: Type 1 DM; T2DM: Type 2 DM; LOFUS: The Lolland-Falster Health Study; HbA1c: Glycated hemoglobin; HDL: High-density lipoprotein cholesterol; UACR: Urine albumin-to-creatinine ratio; ADA: The American Diabetes Association; BMl: Body mass index; RR: Relative risks; MLE: Maximum likelihood estimation; Cl: Confidence intervals; DANHES: The Danish Health Examination Survey; GESUS: The Danish General Suburban Population Study; CGPS: The Copenhagen General Population Study; OGTT: Oral glucose tolerance test; NHANES: National Health and Nutrition Examination Survey; DMRC: Diabetes-related conditions in need of intervention; PCDM: Poorly controlled diabetes; PSCDM: Potentially sub-controlled diabetes; WCDM: Well-controlled diabetes

\section{Acknowledgements}

The Lolland-Falster Health Study (LOFUS), Nykøbing Falster Hospital, Denmark, is a collaboration between Region Zealand, Nykøbing Falster Hospital, and Lolland and Guldborgsund Municipalities. The authors are grateful to LOFUS for making the LOFUS research data available. However, LOFUS bears no responsibility for the analysis or the interpretation conducted within this study.

\section{Authors' contributions}

All authors contributed significantly to the study. RJ provided the LOFUS data. NBR and EL designed the study, interpreted the data, and drafted the manuscript. GN performed the statistical analysis and contributed to the interpretation. CE, AKE, RJ, KR and SB contributed to the interpretation and writing of the manuscript. All authors critically revised and approved the final manuscript.

\section{Funding}

(1) Region Zealand/ University of Copenhagen, (2) Nykøbing Falster Hospital, The Endocrinology Division, (3) Region Zealand research fund, (4) Helsefonden, (5) Professor grants for Elsebeth Lynge. The funding bodies had no role in the design of the study, neither in the collection, analysis, and interpretation of data, nor in the writing of the manuscript.

\section{Availability of data and materials}

Data from the study can be made available via Region Sjaelland following the Danish Data Protection Regulation.

\section{Ethics approval and consent to participate}

Participants provided written informed consent and the Region Zealand's Ethical Committee on Health Research (SJ-421) and the Danish Data Protection Agency (REG-24-2015) approved the study. In the case of abnormal laboratory results, the LOFUS participant was informed by the study nurse by phone and advised to consult his/her general practitioner [9]. All participants were able to check the results of their biochemical analyses on their electronic health records on Sundhed.dk [42].

\section{Consent for publication}

Not applicable.

\section{Competing interests}

The authors declare that they have no competing interests.

\section{Author details}

${ }^{1}$ Center for Epidemiological Research, Nykøbing Falster Hospital,

Strandboulevarden 64, DK-4800 Nykøbing Falster, Denmark. ²Department of Public Health, University of Copenhagen, Copenhagen, Denmark.

${ }^{3}$ Department of Endocrinology, Nykøbing Falster Hospital, Nykøbing Falster, Denmark. ${ }^{4}$ Department of Clinical Biochemistry, Herlev and Gentofte Hospital, Copenhagen, Denmark. ${ }^{5}$ Data and Development Support, Region Zealand, Sorø, Denmark. ${ }^{6}$ Department of Laboratory Medicine, Boston Children's Hospital \& Havard Medical School, Boston, MA, USA.

Received: 8 April 2020 Accepted: 29 October 2020

Published online: 16 November 2020

\section{References}

1. American Diabetes Association. Diagnosis and classification of diabetes mellitus. Diabetes Care. 2009:32:S62-7.

2. Cho NH, Shaw JE, Karuranga S, et al. IDF diabetes atlas: global estimates of diabetes prevalence for 2017 and projections for 2045. Diabetes Res Clin Pract. 2018;138:271-81.

3. Valdés S, Botas P, Delgado E. CadórnigaFD. Mortality risk in Spanish adults with diagnosed diabetes, undiagnosed diabetes, or pre-diabetes. The Asturias study 1998-2004. Re Esp Cardiol. 2009;62:528-34.

4. Wild SH, Smith FB, Lee AJ, Fowkes FG. Criteria for previously undiagnosed diabetes and risk of mortality: 15- year follow-up of the Edinburgh artery study cohort. Diabet Med. 2005;22:490-6.

5. Carstensen B, Kristensen JK, Ottosen P, Borch-Johnsen K, Steering Group of the National Diabetes Register. The Danish National Diabetes Register: trends in incidence, prevalence and mortality. Diabetologia. 2008;51:218796.

6. Jørgensen ME, Ellervik C, Ekholm O, Johansen NB, Carstensen B. Estimates of prediabetes and undiagnosed type 2 diabetes in Denmark: the end of an epidemic or a diagnostic arte- fact? Scand J Public Health. 2018:1-7.

7. Statistics Denmark: https://www.statistikbank.dk/10083. [Accessed 6 January 2020 (data reference item accessed on organization website)].

8. Blaakilde AL, Hansen BH, Olesen LS. Health profile 2017 for region Zealand and municipalities - "How are you?"(in Danish). Sorø: Region Zealand; 2018. (1-6-2019).

9. Jepsen R, Egholm CL, Brodersen J, et al. Lolland-Falster health study: study protocol for a household-based prospective cohort study. Scand J Public Health. 2020;48:382-90.

10. Jepsen $\mathrm{R}$, Wingstrand A, Abild SL, et al. Socio-economic determinants of participation in the Lolland-Falster health study. J Public Health (Berl.). 2019. https://doi.org/10.1007/s10389-019-01095-z.

11. Bowling A. Just one question: if one question works, why ask several? J Epidemiol Community Health. 2005;59:342-5.

12. Danmarks Statistik: https://www.statistikbank.dk/10083 [Accessed 6 January 2020 (data reference item accessed on organization website)].

13. Dansk Selskab for Almen Medicin, Danish Association for General Practice, Clinical Guideline. Type 2 Diabetes - Et metabolisk syndrom. Copenhagen, Denmark: http://vejledninger.dsam.dk/type2 [Accessed 17 December 2019 (data reference item accessed on organization website)].

14. Standards of Medical Care in Diabetes-2019 Abridged for Primary Care Providers: American Diabetes Association Clinical Diabetes 2019; 37:11-34.

15. World Health Organization. In: WHO, editor. Obesity: Preventing and Managing the Global Epidemic. Report of a WHO Consultation on Obesity. Geneva; 1998. Geneva, 3-5 June 1997. WHO Technical Report Series 894.

16. Kautzky-Willer A, Harreiter J, Pacini G. Sex and gender differences in risk, pathophysiology and complications of type 2 diabetes mellitus. Endocr Rev. 2016;37:278-316.

17. DECODE Study Group. Age- and sex-specific prevalences of diabetes and impaired glucose regulation in 13 European cohorts. Diabetes Care. 2003;26: $61-9$.

18. Bertakis KD, Azari R, Helms LJ, Callahan EJ, Robbins JA. Gender differences in the utilization of health care services. J Fam Pract. 2000;49:147-52.

19. Manandhar M, Hawkes S, Buse K, Nosrati E, Magar V. Gender, health and the 2030 agenda for sustainable development. Bull World Health Organ. 2018; 96:644-53.

20. Bergholdt HK, Bathum L, Kvetny J, et al. Study design, participation and characteristics of the Danish general suburban population study. Dan Med J. 2013;60:A4693. 
21. Boucher AB, Adesanya EA, Owei I, et al. Dietary habits and leisure-time physical activity in relation to adiposity, dyslipidemia, and incident Dysglycemia in the pathobiology of Prediabetes in a biracial cohort study. Metabolism. 2015;64:1060-7.

22. Meisinger $\mathrm{C}$, Löwel $H$, Thorand B, Döring A. Leisure time physical activity and the risk of type 2 diabetes in men and women from the general population. The MONICA/KORA Augsburg Cohort Study. Diabetologia. 2005; 48:27-34.

23. Qiu M, Shen W, Song X, et al. Effects of prediabetes mellitus alone or plus hypertension on subsequent occurrence of cardiovascular disease and diabetes mellitus: Iongitudinal study. Hypertension. 2015;65:525-30.

24. Nicolucci A, Cucinotta D, Squatrito S, et al. Clinical and socio-economic correlates of quality of life and treatment satisfaction in patients with type 2 diabetes. Nutr Metab Cardiovasc Dis. 2009;19:45-53.

25. Engström MS, Leksell J, Johansson UB, et al. Health-related quality of life and glycaemic control among adults with type 1 and type 2 diabetes - a nationwide cross-sectional study. Health Qual Life Outcomes. 2019;17:141.

26. Sami W, Ansari T, Butt NS, Hamid MRA. Effect of diet on type 2 diabetes mellitus: a review. Int J Health Sci. 2017;11:65-71.

27. Chang AM, Halter JBA. Aging and insulin secrection. J Physiol Endocrinol Metabol. 2003;284:E7-12.

28. Wu Y, Ding $Y$, Tanaka $Y$, Zhang $W$. Risk factors contributing to type 2 diabetes and recent advances in the treatment and prevention. Int J Med Sci. 2014;11:1185-200.

29. Zhu ZW, Denga FY, Lei SF. Meta-analysis of Atherogenic index of plasma and other lipid parameters in relation to risk of type 2 diabetes mellitus. Prim Care Diabetes. 2015;9:60-7.

30. Stöckl D, Rückert-Eheberg IM, Heier $M$, et al. Regional variability of lifestyle factors and hypertension with prediabetes and newly diagnosed type 2 diabetes mellitus: the population-based kora-f4 and ship-trend studies in Germany. PLoS One. 2016;11:e0156736.

31. Nielson C, Lange T. Blood glucose and heart failure in nondiabetic patients. Diabetes Care. 2005;28:607-11.

32. Tenenbaum A, Fisman EZ. Impaired glucose metabolism in patients with heart failure: pathophysiology and possible treatment strategies. Am J Cardiovasc Drugs. 2004;4:269-80.

33. Vassalotti JA, Li S, Chen SC, Collins AJ. Screening populations at increased risk of CKD: the kidney early evaluation program (KEEP) and the public health problem. Am J Kidney Dis. 2009:107-14.

34. Plantinga LC, Crews DC, Coresh J, et.al. Prevalence of chronic kidney disease in US adults with undiagnosed diabetes or prediabetes. Clin J Am Soc Nephrol 2010; 673-682.

35. Stark Casagrande S, Fradkin JE, Saydah SH, Rust KF, Cowie CC. The prevalence of meeting $\mathrm{A} 1 \mathrm{C}$, blood pressure, and $\mathrm{LDL}$ goals among people with diabetes, 1988-2010. Diabetes Care. 2013;36:2271-9.

36. Yu SH, Kang JG, Hwang YC, et al. Increasing achievement of the target goals for glycemic, blood pressure and lipid control for adults with diagnosed diabetes in Korea. J Diabetes Invest. 2013:4:460-5.

37. Larsen SB, Dalton SO, Schüz J, et al. Mortality among participants and nonparticipants in a prospective cohort study. Eur J Epidemiol. 2012;27:837-45.

38. Blonde L, Aschner P, Bailey C, et al. Gaps and barriers in the control of blood glucose in people with type 2 diabetes. Diab Vasc Dis Res. 2017;14: $172-83$.

39. Glumer C, Carstensen B, Sandbaek A, et al. Inter99 study A Danish diabetes risk score for targeted screening: the Inter99 study. Diabetes Care. 2004;27: $727-33$

40. Gray LJ, Taub NA, Khunti K, et al. The Leicester risk assessment score for detecting undiagnosed type 2 diabetes and impaired glucose regulation for use in a multiethnic UK setting. Diabet Med. 2010;27:887-95.

41. Lau CJ, Pisinger C, Husemoen LL, et al. Effect of general health screening and lifestyle counselling on incidence of diabetes in general population: Inter99 randomised trial. Prev Med. 2016;91:172-9.

42. Sundhed.dk, eHealth in Denmark: https://www.sundhed.dk/borger/service/ om-sundheddk/ehealth-in-denmark/ [Accessed 17 December 2019 (data reference item accessed on organization website)].

\section{Publisher's Note}

Springer Nature remains neutral with regard to jurisdictional claims in published maps and institutional affiliations.

\section{Ready to submit your research? Choose BMC and benefit from:}

- fast, convenient online submission

- thorough peer review by experienced researchers in your field

- rapid publication on acceptance

- support for research data, including large and complex data types

- gold Open Access which fosters wider collaboration and increased citations

- maximum visibility for your research: over $100 \mathrm{M}$ website views per year

At $\mathrm{BMC}$, research is always in progress.

Learn more biomedcentral.com/submissions 